\title{
LOS BENEFICIOS DE UNA CORRECTA EVALUACIÓN FORMATIVA EN EL AUTOAPRENDIZAJE DE LOS ALUMNOS
}

\author{
THE BENEFITS OF A CORRECT FORMATIVE ASSESSMENT IN \\ STUDENTS 'SELF-LEARNING
}

Mónica Azpilicueta Amorín

\begin{abstract}
Science teachers in the Middle Years Program (MYP) are often dissatisfied with the grades achieved by their students. Hence, the objective of this work is to demonstrate that good planning and implementation of formative assessment based on correct feedback favors students' self-learning, translating into a progressive increase in their grades.

From the numerous bibliography on the matter about the benefits that formative assessment provides to students, this work demonstrates the benefits that the feedback proposed by the authors Arthur L. Costa and Robert J. Gamston brings to students who study Science in the last year of the MYP.

Analyzed / compared the data of the qualifications obtained before and after the implementation of the feedback, it is demonstrated that in those aspects of the evaluation that require more skill and training, the qualification of the subject has risen up to two points thanks to the formative assessment.

Demonstrating that, despite the difficulty involved in making a good formative evaluation a reality in the classroom, based on quality feedback, the teaching / learning process is enhanced, translating not only into an increase in grades, but also into teaching lasting for the student.
\end{abstract}

Key words: MYP, Formative evaluation, Science, feedback and self-directed learners.

\section{RESUMEN}

A menudo los docentes de Ciencias del Programa de los Años Intermedios (PAI) no se encuentran satisfechos con las calificaciones obtenidas por sus alumnos. De ahí que el objetivo de este trabajo sea el demostrar que una buena planificación e implementación de la evaluación formativa basada en una correcta retroalimentación favorece el autoaprendizaje de los alumnos traduciéndose en un aumento progresivo de sus calificaciones.

De la numerosa bibliografía que hay al respecto sobre los beneficios que la evaluación formativa proporciona a los alumnos, este trabajo demuestra los beneficios que la retroalimentación propuesta por los autores Arthur L. Costa y Robert J. Gamston aporta a los alumnos que cursan Ciencias en el último curso del PAI.

Analizados/comparados los datos de las calificaciones obtenidas antes y después de la implementación de la retroalimentación, se demuestra que en aquellos aspectos de la evaluación que requieren más destreza y entrenamiento, han llegado a subir hasta dos puntos la calificación de la asignatura gracias a la evaluación formativa.

Demostrando que, pese a la dificultad que conlleva hacer realidad en el aula, una buena evaluación formativa, basada en una retroalimentación de calidad, se potencia el proceso de enseñanza/aprendizaje traduciéndose, no solo en un aumento en las calificaciones, sino en una enseñanza perdurable para el alumno.

Palabras clave: PAI, Evaluación formativa, Ciencias, retroalimentación y autoaprendizaje. 
Fecha de recepción: 24 de septiembre de 2020.

Fecha de aceptación: 4 de diciembre de 2020. 


\section{INTRODUCCIÓN}

Las Ciencias corresponde a uno de los ocho grupos de asignaturas (figura 1) en los que se organizan las disciplinas en el Programa de los Años Intermedios (PAI) del Bachillerato Internacional (IB), programa que incluye a los alumnos de edades comprendidas entre los 11 y los 16 años de edad.

Figura 1: Modelo del Programa de los Años Intermedios (PAI)

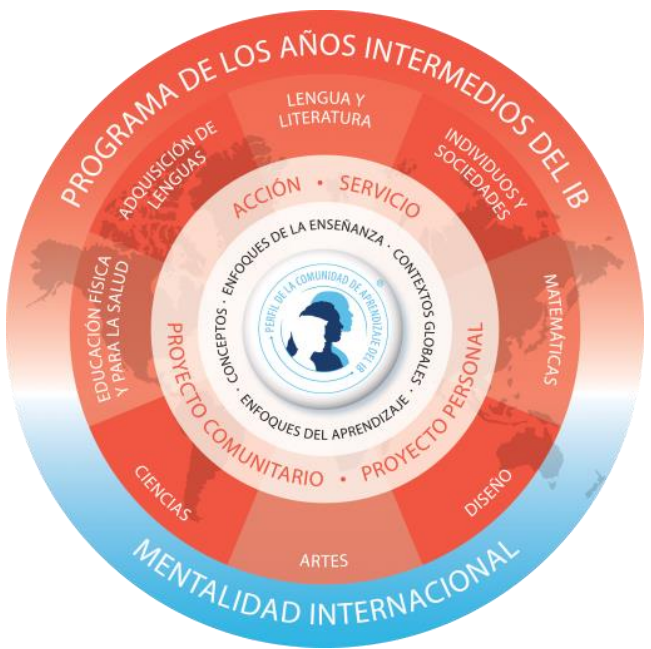

Fuente: Organización Bachillerato Internacional

Dentro del grupo de las Ciencias, se engloban las asignaturas de Biología, Geología, Física y Química. En concreto, el trabajo que nos ocupa se realizó en la asignatura de Física y Química del último curso del programa: PAI 5.

El aspecto más positivo que implica impartir una asignatura de Ciencias en PAI es, sin ninguna duda, el hecho de que la metodología de enseñanza debe ser mayoritariamente práctica, donde son los alumnos los que van construyendo su conocimiento a partir de las experiencias de aprendizaje que el docente les van planteando a lo largo de las clases.

Sin embargo, este aprendizaje de calidad puede verse afectado negativamente por una mala planificación curricular o un ineficaz diseño en la evaluación debido a diversos factores como puede ser la falta de tiempo o de experiencia.

Si atendemos al objetivo de nuestro trabajo, es muy habitual que, al comienzo, el diseño de la evaluación no sea el más adecuado para poder conseguir los mejores resultados posibles por parte de todos los alumnos, centrándose exclusivamente los docentes en la elaboración de una correcta evaluación sumativa (debido a la repercusión que ésta tiene directamente sobre la calificación final de la disciplina).

De hecho, la fuerza que presentan las tareas de tipo examen o pruebas escritas sigue siendo exageradamente mayor que otro tipo de tareas como proyectos, tareas con rúbricas, presentaciones orales, actividades centradas en rúbricas... (Valle, Menéndez, Manso, Garrido, \& Thoilliez, 2017). Requiriendo, estas últimas, de una práctica guiada por parte del docente debido a que supone el desarrollo del pensamiento crítico de los alumnos y la evolución de habilidades como la de comunicación y autogestión para que sea eficaz su aprendizaje. 
Sin embargo, no se suele prestar la misma atención (al menos al principio) a la evaluación formativa, evaluación que nos permite trabajar todas estas habilidades de manera progresiva a través de los comentarios aportados por el docente y la madurez que va adquiriendo el alumno gracias a su experiencia y al aprendizaje a partir de sus "errores".

Pero, si reflexionamos sobre el impacto que la evaluación formativa realmente tiene en los alumnos, nos daremos cuenta de su importancia. No solo porque va a hacer que el aprendizaje del alumno sea mucho más duradero y enriquecedor, sino que, también ayudará al alumno a progresar en la consecución de sus logros y en su motivación.

En la evaluación del PAI debemos atender por igual a dos tipos de evaluación: formativa (donde el docente puede obtener información sobre las necesidades que requieren los alumnos, planificar los siguientes pasos en función de la información obtenida, ofrecerle a los alumnos actividades de aprendizaje con objetivos marcados para darles retroalimentación y poder así guiarles en su proceso de aprendizaje) y sumativa (la cual determina el nivel de competencia que presentan nuestros alumnos en un momento determinado en su proceso de aprendizaje, obteniéndose los resultados a partir de diferentes tareas sumativas que se realizan en un periodo determinado) y así debe constar en la política de evaluación de colegio (IB, El programa de los Años Intermedios: de los principios a la práctica, para uso a partir de septiembre de 2014 o enero de 2015, 2014).

La realización de las tareas formativas debe permitir a todos los alumnos descubrir y mejorar sus habilidades necesarias para construir un aprendizaje de calidad en Ciencias y poder obtener los mejores resultados posibles en los diferentes aspectos (clasificados a continuación como i, ii, iii, iv, v) de los cuatro criterios de los que compone la asignatura de Ciencias (IB, Guía de Ciencias para uso a partir de septiembre de 2014 o enero de 2015, 2014) :

\section{Criterio A / Conocimiento y comprensión}

i. Explicar conocimientos científicos

ii. Aplicar conocimientos y comprensión científicos para resolver problemas en situaciones tanto conocidas como desconocidas

iii. Analizar y evaluar información para emitir juicios con base científica

\section{Criterio B / Indagación y Diseño}

i. Explicar un problema o una pregunta que se quieren comprobar mediante una investigación científica

ii. Formular una hipótesis comprobable y explicarla mediante un razonamiento científico

iii. Explicar cómo manipular las variables y explicar cómo se obtendrán los datos

iv. Diseñar investigaciones científicas

\section{Criterio C / Procesamiento y evaluación}

i. Presentar los datos obtenidos y transformados

ii. Interpretar los datos y explicar los resultados mediante un razonamiento científico

iii. Evaluar la validez de una hipótesis según el resultado de la investigación científica

iv. Evaluar la validez del método

v. Explicar las mejoras o ampliaciones del método

\section{Criterio D / Reflexión sobre el impacto de la Ciencia}

i. Explicar de qué maneras se aplica y se utiliza la ciencia para abordar una cuestión o un problema concreto 
ii. Discutir y evaluar las diversas implicaciones del uso de la ciencia y su aplicación a la resolución de una cuestión o un problema concretos

iii. Aplicar lenguaje científico de forma eficaz

iv. Documentar el trabajo de otras personas y las fuentes de información que utilicen

Analizando en profundidad los aspectos de los cuatro criterios que acabamos de detallar, se puede deducir que una enseñanza y una evaluación tradicional no permitiría a los alumnos poder alcanzar las bandas más altas de calificación en los mismos ${ }^{1}$.

Sobre todo, hay dos criterios, el $\mathrm{B}$ y el C, que son inminentemente prácticos, que requieren que el alumno se dé cuenta por sí mismo de cómo se deben realizar los diferentes pasos del método científico para poder diseñar experimentos y cómo es la manera más apropiada de presentar y analizar los datos que ellos mismos van obteniendo en sus experimentaciones. Estas acciones requieren un correcto diseño de tareas formativas que adjunten comentarios del docente personalizados para comprender perfectamente cómo debe responder el alumno y de qué manera lo que ha aprendido lo puede extrapolar a escenarios desconocidos para él.

Por otro lado, en los criterios A y D los alumnos tampoco podrían alcanzar grandes calificaciones si el docente no les proporcionara una retroalimentación adecuada, positiva y motivadora, ya que requieren mucho más que una simple memorización de una materia o de un procedimiento para resolver problemas. La resolución de tareas de estos criterios contiene un factor muy importante de destreza de habilidades tales como pensamiento crítico, autoestima, autogestión y comunicativas muy fuerte.

Si se focaliza la evaluación, desde un punto de vista formativo, no solo mejoraremos el aprendizaje de nuestros alumnos, sino también organizaremos la metodología de enseñanza / aprendizaje con el fin de adaptar las condiciones pedagógicas atendiendo a la diversidad en el aula (Franco, 2012) haciendo que los alumnos sean realmente los constructores de su aprendizaje.

Por ello, este estudio se centró en la investigación de la evolución de los resultados de los alumnos en la asignatura de Física y Química (Ciencias) antes y después de la aplicación de la retroalimentación propuesta, de manera general por los autores Costa y Gamston (Costa \& Garmston, 2013) en la evaluación formativa de dicha asignatura a lo largo de cuatro cursos escolares, desde 2016/2017 hasta 2019/2020.

Según dichos autores, dentro de un gran abanico de posibilidades a la hora de ofrecerles retroalimentación a los alumnos en la evaluación formativa, hay cinco procedimientos (ordenados en orden decreciente de éxito) que son los que mejores resultados les proporciona a los alumnos:

1. Interrogatorio reflexivo

2. Aporte de datos sin prejuicios

3. Inferencias, causalidad e interpretaciones

4. Opiniones y preferencias personales

5. Evaluaciones y juicios

Este estudio pretende demostrar cómo efectivamente la aportación de una correcta retroalimentación a los alumnos basada en los cinco procedimientos mencionados anteriormente, les permite ir construyendo su propio aprendizaje, adquiriendo las herramientas necesarias para la

\footnotetext{
${ }^{1}$ Los cuatro criterios de la asignatura pueden alcanzar como máximo un valor de 8 puntos. Las rúbricas en las que se detallan las acciones que debe desarrollar el alumno para conseguir cada uno de los niveles de logro vienen detallados en la propia guía de Ciencias para el último curso del PAI (IB, Guía de Ciencias para uso a partir de septiembre de 2014 o enero de 2015, 2014).
} 
elaboración exitosa de las diferentes tareas de Física y Química (Ciencias) aumentando la autoestima de los alumnos y, por tanto, sus ganas de aprender y conocer el mundo que les rodea.

\section{METODOLOGÍA}

Tras la publicación de la nueva guía de Ciencias del proyecto: "Programa de los Años Intermedios, el siguiente capítulo" (en el curso escolar 2014/2015), se realizaron los cambios necesarios en la planificación tanto en la metodología de enseñanza como en la evaluación de la asignatura de Física y Química en último curso del programa: PAI 5 de nuestro centro.

Los cambios fueron bastante drásticos en la evaluación, no tanto en la metodología, por lo que se tuvo que reestructurar toda la evaluación de la asignatura en dicho curso. Durante los dos primeros cursos escolares tras este cambio, los esfuerzos se centraron en la comprensión y aplicación adecuada de los nuevos criterios ya que pasaron de ser seis criterios a cuatro (figura 2) y en el diseño de calidad de tareas sobre todo sumativas para adaptarlas a la nueva guía de Ciencias.

Figura 2: Cambio de los criterios de Ciencias en el PAI
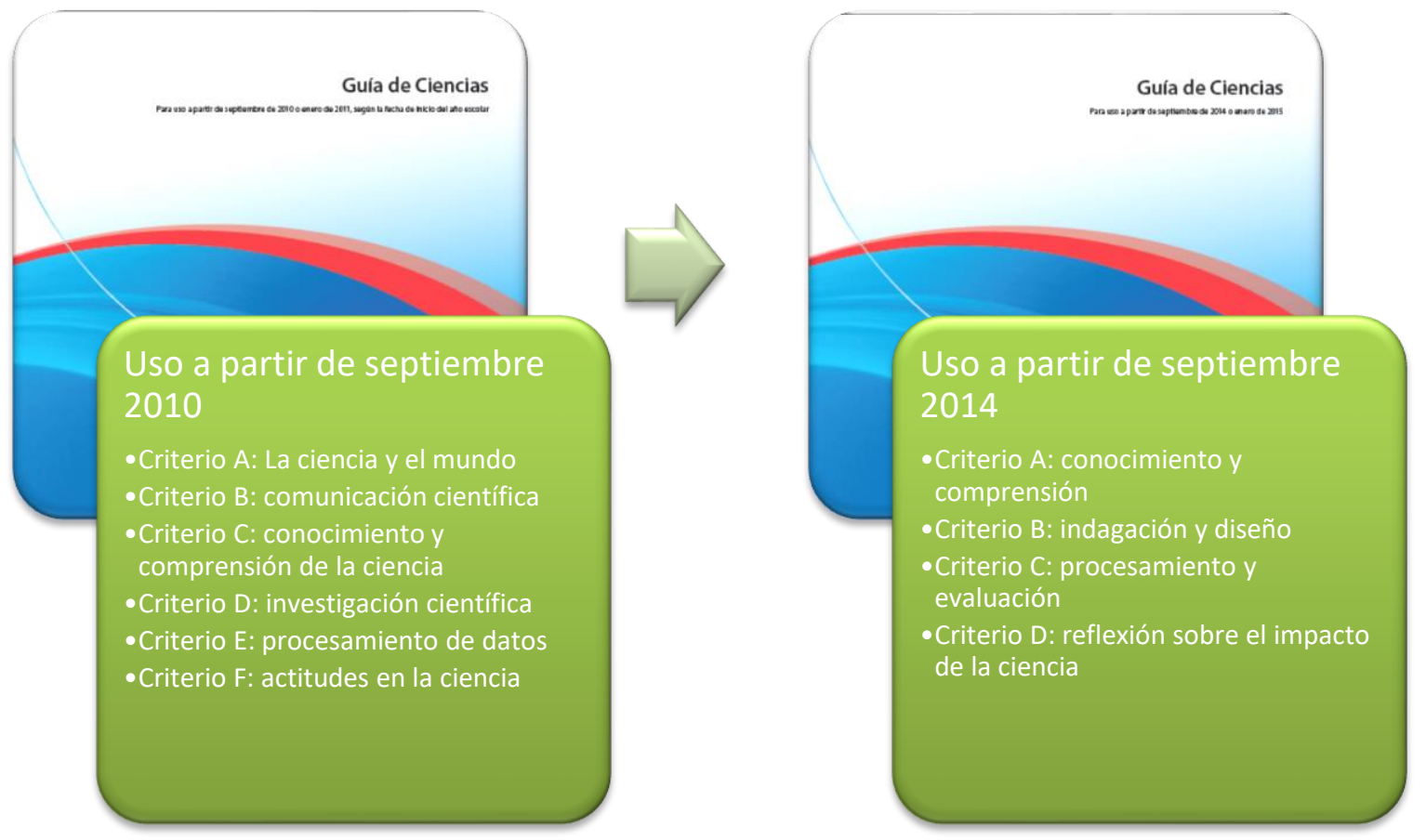

Fuente: Elaboración propia

Sin embargo, fue en el curso escolar 2016/2017, cuando a partir de la experiencia docente y las recomendaciones aportadas por Costa y Garmston (Costa \& Garmston, 2013) así como la reflexión aportada por los propios alumnos de los cursos escolares anteriores, se rediseñó la evaluación formativa que se estaba llevando a cabo en la asignatura de Física y Química en PAI 5 de nuestro centro, con el fin de poder analizar el efecto que realmente tiene lugar en la calificación de la asignatura el que se lleve a cabo en el aula una buena evaluación formativa basada en la retroalimentación (figuras 3 y tabla 1 ). 
Figura 3: Línea del tiempo del proceso del estudio

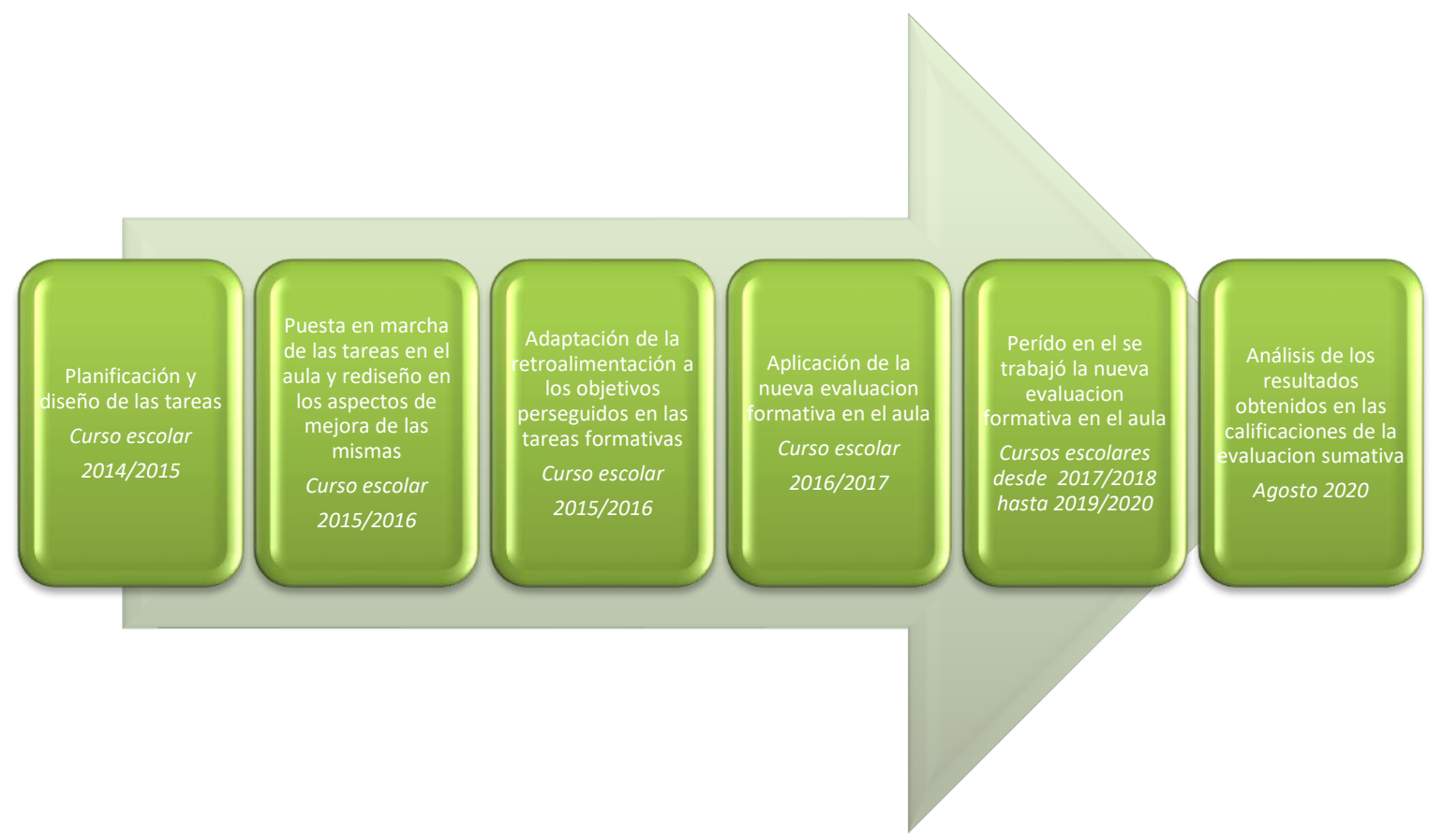

Fuente: Elaboración propia

Tabla 1: Mejoras de la evaluación formativa en la asignatura de Física y Química (Ciencias) en PAI 5 basadas en los procedimientos de retroalimentación propuestos por los autores Costa y

\section{Garmston}

\begin{tabular}{|c|c|c|c|}
\hline $\begin{array}{l}\text { Tipo de } \\
\text { retroalimentación } \\
\text { aportada a los } \\
\text { alumnos }\end{array}$ & $\begin{array}{l}\text { Breve descripción } \\
\text { del tipo de } \\
\text { retroalimentación } \\
\text { aportada por los } \\
\text { autores }\end{array}$ & $\begin{array}{l}\text { Ejemplos de la retroalimentación } \\
\text { aportada a los alumnos }\end{array}$ & $\begin{array}{c}\text { Enfoque de } \\
\text { aprendizaje }{ }^{2} \text { del IB } \\
\text { potenciado a través } \\
\text { del tipo de } \\
\text { retroalimentación } \\
\text { empleado }\end{array}$ \\
\hline 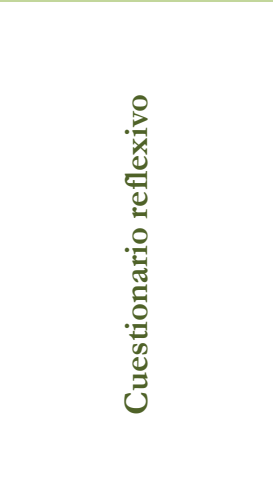 & $\begin{array}{l}\text { Formulación de una } \\
\text { serie de preguntas } \\
\text { guiadas por el } \\
\text { docente que vaya } \\
\text { encaminando al } \\
\text { alumno a obtener } \\
\text { los resultados } \\
\text { deseados a partir de } \\
\text { sus propias } \\
\text { indagaciones y } \\
\text { reflexiones. }\end{array}$ & $\begin{array}{l}\text { La propia metodología del PAI permite } \\
\text { desarrollar este tipo de } \\
\text { retroalimentación diariamente en el aula. } \\
\text { Pero, a nivel individual o en parejas, se } \\
\text { desarrollaron semanalmente (antes de la } \\
\text { evaluación sumativa) tareas en las que } \\
\text { los alumnos recibían este tipo de } \\
\text { comentarios, donde, a partir de } \\
\text { preguntas se les iba guiando para poder } \\
\text { dar solución a los retos planteados. }\end{array}$ & $\begin{array}{l}\text { Habilidades de: } \\
\text { - Reflexión } \\
\text { - Organización } \\
\text { - Gestión de la } \\
\text { información } \\
\text { - Alfabetización } \\
\text { Mediática } \\
\text { - Pensamiento crítico } \\
\text { - Pensamiento } \\
\text { creativo } \\
\text { - Transferencia }\end{array}$ \\
\hline
\end{tabular}

2 "A través de los enfoques de aprendizaje los alumnos desarrollan habilidades pertinentes a todo el currículo y que nos ayudan a aprender a aprender" (IB, El programa de los Años Intermedios: de los principios a la práctica, para uso a partir de septiembre de 2014 o enero de 2015, 2014) 


\begin{tabular}{|c|c|c|c|}
\hline 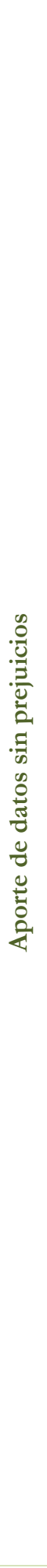 & $\begin{array}{l}\text { El docente presenta } \\
\text { una serie de datos } \\
\text { que son compartidos } \\
\text { con los alumnos y se } \\
\text { les anima a que } \\
\text { reflexionen e } \\
\text { interpreten junto } \\
\text { con los datos } \\
\text { aportados por la } \\
\text { clase para que } \\
\text { apliquen esta } \\
\text { interpretación en sus } \\
\text { trabajos futuros. }\end{array}$ & 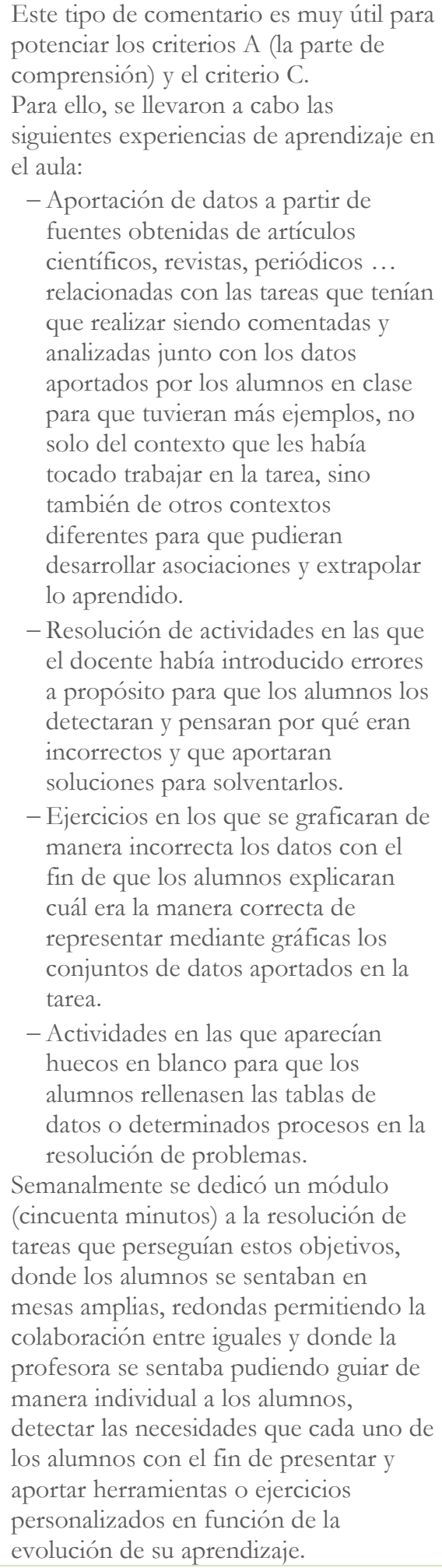 & $\begin{array}{l}\text { Habilidades de: } \\
\text { - Reflexión } \\
\text { - Comunicación } \\
\text { - Gestión de la } \\
\text { información } \\
\text { - Pensamiento crítico } \\
\text { - Pensamiento } \\
\text { creativo } \\
\text { - Transferencia }\end{array}$ \\
\hline
\end{tabular}




\begin{tabular}{|c|c|c|c|}
\hline 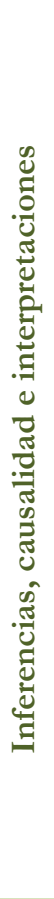 & $\begin{array}{l}\text { Esta } \\
\text { retroalimentación no } \\
\text { es tan enriquecedora } \\
\text { para los alumnos ya } \\
\text { que depende mucho } \\
\text { de la interpretación } \\
\text { y/o de las } \\
\text { conexiones que haga } \\
\text { el docente, por lo } \\
\text { que los alumnos } \\
\text { solo perciben el } \\
\text { punto de vista del } \\
\text { profesor. }\end{array}$ & $\begin{array}{l}\text { Se aplicó para los cuatro criterios, pero } \\
\text { mayoritariamente para el criterio A (para } \\
\text { la parte de la comprensión), criterio B y } \\
\text { criterio D. } \\
\text { Ofreciendo ejemplos de cómo se } \\
\text { deberían de contestar diferentes tareas, } \\
\text { en función de los términos de } \\
\text { instrucción }{ }^{3} \text {, para que se pudieran } \\
\text { alcanzar las bandas más altas de los } \\
\text { diferentes aspectos de los criterios y de } \\
\text { qué manera se podría transferir lo } \\
\text { aprendido a otros casos desconocidos. } \\
\text { De esta forma, los alumnos observaron } \\
\text { otros modos diferentes de proceder a la } \\
\text { hora de trabajar los diferentes conceptos } \\
\text { de la asignatura, a reflexionar sobre } \\
\text { otros puntos de vista diferentes a los } \\
\text { suyos, de cómo se puede proceder para } \\
\text { resolver las distintas tareas propuestas y, } \\
\text { muy importante, cómo se pueden } \\
\text { transferir los conocimientos dentro de la } \\
\text { misma asignatura o de manera } \\
\text { interdisciplinar. }\end{array}$ & $\begin{array}{l}\text { Habilidades de: } \\
\text { - Reflexión } \\
\text { - Gestión de la } \\
\text { información } \\
\text { - Pensamiento crítico } \\
\text { - Transferencia }\end{array}$ \\
\hline 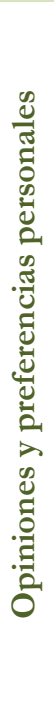 & $\begin{array}{l}\text { Debido a que en } \\
\text { este tipo de } \\
\text { retroalimentación el } \\
\text { profesor aporta sus } \\
\text { opiniones o gustos, } \\
\text { este tipo de } \\
\text { comentarios } \\
\text { solamente les } \\
\text { permite a los } \\
\text { alumnos reforzar sus } \\
\text { acciones, pero } \\
\text { realmente no les } \\
\text { permite mejorar su } \\
\text { capacidad de } \\
\text { autoaprendizaje } \\
\text { porque está basado } \\
\text { en la perspectiva del } \\
\text { profesor. }\end{array}$ & $\begin{array}{l}\text { Siempre que se ofrecía un comentario } \\
\text { de retroalimentación, para cualquier } \\
\text { criterio, se aportaban comentarios de } \\
\text { mejora, pasos que podrían seguir los } \\
\text { alumnos para poder mejorar su } \\
\text { aprendizaje. Independientemente del } \\
\text { criterio que se estuviese trabajando. } \\
\text { Este tipo de comentarios les permitió a } \\
\text { los alumnos organizarse y reflexionar en } \\
\text { función de su situación real en el } \\
\text { proceso de aprendizaje, lugar en el que } \\
\text { se encontraban y a reflexionar sobre su } \\
\text { situación real en el proceso de } \\
\text { aprendizaje. }\end{array}$ & $\begin{array}{l}\text { Habilidades de: } \\
\text { - Reflexión } \\
\text { - Organización }\end{array}$ \\
\hline
\end{tabular}

\footnotetext{
3 "Los términos de instrucción están incorporados en los objetivos específicos y los criterios de evaluación de cada grupo de las asignaturas. Estos términos son empleados por los docentes para dar instrucciones, hacer preguntas a los alumnos, plantear problemas y obtener respuestas de la clase" (IB, El programa de los Años Intermedios: de los principios a la práctica, para uso a partir de septiembre de 2014 o enero de 2015, 2014).
} 


\begin{tabular}{|c|c|c|c|}
\hline 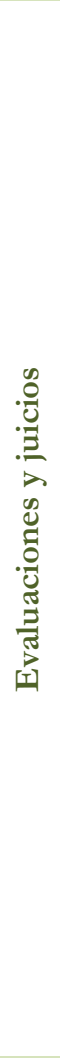 & $\begin{array}{l}\text { La retroalimentación } \\
\text { cuantitativa a } \\
\text { menudo se piensa } \\
\text { que es muy buena } \\
\text { para el alumno ya } \\
\text { que sabe en dónde } \\
\text { se encuentra } \\
\text { realmente. Sin } \\
\text { embargo, puede } \\
\text { provocar el efecto } \\
\text { contrario } \\
\text { transmitiéndole al } \\
\text { alumno que, al final, } \\
\text { la opinión del } \\
\text { profesor es lo que } \\
\text { cuenta. Por eso este } \\
\text { tipo de } \\
\text { retroalimentación es } \\
\text { considerado como el } \\
\text { que menos ayuda } \\
\text { aporta a los alumnos } \\
\text { en su } \\
\text { autoaprendizaje } \\
\text { dentro de los cinco } \\
\text { procedimientos que } \\
\text { evaluaron Costa y } \\
\text { Garmston. }\end{array}$ & $\begin{array}{l}\text { Dentro de la planificación de la } \\
\text { evaluación formativa, se diseñaron } \\
\text { actividades y tareas en todas las } \\
\text { unidades del curso con un esquema de } \\
\text { calificación para que fueran los propios } \\
\text { alumnos los que se autocalificaran o } \\
\text { calificaran el trabajo de otros } \\
\text { compañeros a partir de rúbricas claras. } \\
\text { Estas acciones les permitieron a los } \\
\text { alumnos ser más conscientes de la } \\
\text { calificación que tendrían sus tareas y, en } \\
\text { muchos casos, de lo que se esperaba que } \\
\text { respondieran en función de los términos } \\
\text { de instrucción. } \\
\text { Dichas actividades/tareas se proponían } \\
\text { con el tiempo suficiente para que los } \\
\text { alumnos pudiesen reaccionar a la hora } \\
\text { de preparar las tareas sumativas } \\
\text { sirviéndoles como punto de partida en la } \\
\text { reflexión de su aprendizaje y a poder } \\
\text { controlar su posible estado de estrés o } \\
\text { ansiedad al ser conocedor de la situación } \\
\text { real, respecto a su aprendizaje, en la que } \\
\text { se encuentra. }\end{array}$ & $\begin{array}{l}\text { Habilidades de: } \\
\text { - Reflexión } \\
\text { - Afectivas }\end{array}$ \\
\hline
\end{tabular}

Fuente: Elaboración propia

Al poner en acción la retroalimentación de modo que se le informa al alumno la calificación obtenida en su trabajo, justificándole el por qué ha obtenido dicha calificación, comentándole qué le falta para poder acceder a la banda alta y felicitándole por los logros conseguidos, animándole a seguir superándose, lo que conseguimos es promover la metacognición del alumno volviéndose más autónomo y consciente del lugar en el que se encuentra en su aprendizaje y hasta dónde puede llegar (Sadler, 2010).

En nuestro centro, a lo largo de un curso escolar, generamos un boletín de calificaciones cada tres meses. En dicho boletín se puede observar la calificación que el alumno ha obtenido en cada uno de los cuatro criterios de cada asignatura. De esta forma, el alumno y las familias pueden observar y analizar la calificación que ha obtenido en ese trimestre en los cuatro criterios y la nota global que consigue en el trimestre en cada asignatura.

El hecho de informar a las familias sobre la evaluación de los alumnos (en cada una de las tareas y trimestralmente) ha sido vital para poder alcanzar el éxito real que ha tenido este estudio, puesto que les proporciona información sobre las habilidades que presentan sus hijos, así como la situación real de lo que saben y lo que deben mejorar. Resultando positivamente el apoyo de las familias, en la enseñanza /aprendizaje de los alumnos desde edades tempranas (Osorio Sánchez \& Löpez Mendoza, 2014).

Relacionado con la asignatura de Física y Química, en el boletín trimestral mencionado anteriormente, la nota obtenida, en cada criterio, refleja el desarrollo que ha obtenido el alumno a lo largo de los tres meses, gracias a las tareas formativas, a partir de las cuales se les brinda un feedback (comprendido por comentarios de mejora, y datos numéricos) a cada uno de los alumnos 
con el fin de potenciar las habilidades necesarias de todos los alumnos para que puedan elaborar sin problema las tareas sumativas las cuales son calificadas cuantitativamente y reflejadas en los boletines trimestrales.

Los datos empleados en este trabajo son, precisamente, las calificaciones cuantitativas de los cuatro criterios de Ciencias que han obtenido los alumnos de PAI 5 desde el curso escolar 2016/2017 hasta el curso 2019/2020 (en este último curso, debido a la particular situación derivada de la COVID-19, solamente se han incluido los datos de los dos primeros trimestres ya que fueron calificados íntegramente en el centro, por lo que los datos empelados reflejan las mismas condiciones de trabajo que las de los cursos anteriores empleados en el estudio).

Asimismo, aclarar que, se han empezado a tomar en consideración los datos del curso escolar 2016/2017 ya que la nueva guía de Ciencias se puso en marcha en el curso escolar 2014/2015, por lo que la nueva metodología de evaluación (que es lo que estamos analizando en este trabajo) no se estabilizó al cien por cien hasta el curso escolar en el que comienza nuestro estudio.

Es importante destacar que la docente de la asignatura en la que se centra este estudio (Física y Química) ha sido la misma a lo largo del periodo del estudio por lo que el criterio del empleo de las rúbricas y de las puntuaciones de las tareas ha sido homogéneo desde el comienzo hasta el fin.

Por último, comentar que, la asignatura de Física y Química en nuestro centro es una asignatura obligatoria para todos aquellos alumnos que quieran cursar PAI, por lo que no resulta igual de motivadora para todos los alumnos que la cursan.

Los datos de los cuatro criterios de Ciencias fueron analizados a través del programa IBM SPSS Statistics Visor con el fin de demostrar si realmente existió o no un cambio positivo en las medias de las calificaciones de los alumnos de Física y Química, tras haber realizado el cambio en la evaluación formativa haciendo hincapié en la retroalimentación aportada por la docente a los alumnos.

\section{RESULTADOS}

\subsection{MEDIAS}

Con el fin de comprobar que la calificación obtenida en los cuatro criterios de Física y Química (Ciencias) del PAI dependen de una evaluación formativa de calidad, basada en una retroalimentación motivadora, se han analizado las calificaciones de cuatro cursos escolares consecutivos.

En total, el estudio se centra en el empleo de 544 datos para cada criterio (A, B, C y D), de los cuales 137 corresponden al curso escolar 2016/2017, 159 al curso escolar 2017/2018, 136 al curso escolar 2018/2019 y, por último, 112 al curso escolar 2019/2020 (se pueden ver detalladamente en el anexo).

Queremos saber si las calificaciones medias de cada uno de los cuatro criterios son iguales en los cuatro años en los que se ha realizado el estudio.

Para verificar si realmente las calificaciones aumentaron tras la implementación de la nueva evaluación formativa o no, realizamos el siguiente contraste de hipótesis: 


$$
\mathrm{H}_{0} \equiv \mu_{1}=\mu_{2}=\mu_{3}=\mu_{4}=\mu \quad \text { vs } \quad \mathrm{H}_{1} \equiv \mu_{\mathrm{i}} \neq \mu_{\mathrm{j}} \text { para algún } \mathrm{i} \neq \mathrm{j}
$$

Por lo tanto, nuestra hipótesis inicial es que no hay diferencias en las medias de los cuatro criterios de Física y Química (Ciencias) en los cuatro cursos escolares estudiados frente a la alternativa de que al menos una media difiere de la otra.

Las medias de las calificaciones de los cuatro criterios de la asignatura obtenidas a lo largo del intervalo de estudio se pueden apreciar en la tabla 2.

Tabla 2: Medias de los cuatro criterios de Ciencias (A, B, C y D) a lo largo de los cursos escolares analizados (2016/2017-2019/2020)

\begin{tabular}{|c|c|c|c|c|}
\hline Curso Escolar & $\begin{array}{c}\text { Calificación } \\
\text { Criterio A }\end{array}$ & $\begin{array}{c}\text { Calificación } \\
\text { Criterio B }\end{array}$ & $\begin{array}{c}\text { Calificación } \\
\text { Criterio C }\end{array}$ & $\begin{array}{c}\text { Calificación } \\
\text { Criterio D }\end{array}$ \\
\hline \multicolumn{5}{|l|}{$2016 / 2017$} \\
\hline Media & 4,09 & 4,77 & 4,10 & 5,16 \\
\hline $\mathrm{N}$ & 137 & 137 & 137 & 137 \\
\hline Desviación estándar & 2,133 & 2,118 & 1,960 & 2,105 \\
\hline Mínimo & 1 & 0 & 1 & 0 \\
\hline Máximo & 8 & 8 & 8 & 8 \\
\hline Error estándar de la media & 0,182 & 0,181 & 0,167 & 0,180 \\
\hline \multicolumn{5}{|l|}{$2017 / 2018$} \\
\hline Media & 4,94 & 4,82 & 4,42 & 5,86 \\
\hline & 159 & 159 & 159 & 159 \\
\hline Desviación estándar & 1,895 & 1,713 & 1,847 & 1,204 \\
\hline Mínimo & 1 & 1 & 1 & 2 \\
\hline Máximo & 8 & 8 & 8 & 8 \\
\hline Error estándar de la media & 0,150 & 0,136 & 0,146 & 0,095 \\
\hline \multicolumn{5}{|l|}{$2018 / 2019$} \\
\hline Media & 4,13 & 5,35 & 5,25 & 5,97 \\
\hline & 136 & 136 & 136 & 136 \\
\hline Desviación estándar & 1,644 & 1,744 & 2,018 & 1,780 \\
\hline Mínimo & 1 & 0 & 0 & 0 \\
\hline Máximo & 8 & 8 & 8 & 8 \\
\hline Error estándar de la media & 0,141 & 0,150 & 0,173 & 0,153 \\
\hline \multicolumn{5}{|l|}{$2019 / 2020$} \\
\hline Media & 4,08 & 6,58 & 5,58 & 6,03 \\
\hline & 112 & 112 & 112 & 112 \\
\hline Desviación estándar & 1,831 & 1,249 & 1,625 & 1,311 \\
\hline Mínimo & 1 & 1 & 1 & 3 \\
\hline Máximo & 8 & 8 & 8 & 8 \\
\hline Error estándar de la media & 0,173 & 0,118 & 0,154 & 0,124 \\
\hline \multicolumn{5}{|l|}{ Total } \\
\hline Media & 4,34 & 5,30 & 4,79 & 5,75 \\
\hline $\mathrm{N}$ & 544 & 544 & 544 & 544 \\
\hline Desviación estándar & 1,920 & 1,879 & 1,962 & 1,670 \\
\hline Mínimo & 1 & 0 & 0 & 0 \\
\hline Máximo & 8 & 8 & 8 & 8 \\
\hline Error estándar de la media & 0,082 & 0,081 & 0,084 & 0,072 \\
\hline
\end{tabular}

Fuente: IBM SPSS Statistics Visor

Como se puede apreciar en la tabla 2, la calificación media de los cuatro criterios (A, B, C y D) aumentó a medida que fueron transcurriendo los cursos escolares (a excepción del criterio A en el curso escolar 2019/2020, cuya calificación media fue prácticamente idéntica a la obtenida antes de la implementación de la evaluación formativa). 
Además, si atendemos a las medias totales (de todos los cursos estudiados) el criterio A es el criterio con la calificación más baja: 4,34, seguido del criterio C $(4,79)$, mientras que los criterios con mayor puntuación obtenida fueron el criterio $\mathrm{B}$ con 5,30 y por último el $\mathrm{D}$ con 5,75.

Para dar respuesta a nuestro estudio, nos interesaba conocer la diferencia que hay entre la calificación media obtenida de cada criterio en el curso escolar previo a la implementación de la evaluación formativa: 2016/2017 y las calificaciones medias en cada uno de los cursos analizados tras dicha implementación (tabla 3)

Tabla 3: Diferencias de las medias de los cuatro criterios (A, B, C y D) antes y después de la mejora en la evaluación formativa (curso escolar 2016/2017)

\begin{tabular}{|c|c|c|c|c|}
\hline $\begin{array}{l}\text { Curso } \\
\text { escolar }\end{array}$ & $\begin{array}{l}\text { Diferencia de la } \\
\text { calificación en el } \\
\text { criterio A }\end{array}$ & $\begin{array}{c}\text { Diferencia de la } \\
\text { calificación en el } \\
\text { criterio B }\end{array}$ & $\begin{array}{c}\text { Diferencia de la } \\
\text { calificación en el } \\
\text { criterio C }\end{array}$ & $\begin{array}{l}\text { Diferencia de la } \\
\text { calificación en el } \\
\text { criterio D }\end{array}$ \\
\hline $2017 / 2018$ & $4,94-4,09=\mathbf{0 , 8 5}$ & $4,82-4,77=0,05$ & $4,42-4,10=0,32$ & $5,86-5,16=\mathbf{0 , 7 0}$ \\
\hline $2018 / 2019$ & $4,13-4,09=\mathbf{0 , 0 4}$ & $5,35-4,77=0,58$ & $5,25-4,10=1,15$ & $5,97-5,16=0,81$ \\
\hline $2019 / 2020$ & $4,08-4,09=-0,01$ & $6,58-4,77=1,81$ & $5,58-4,10=1,48$ & $6,03-5,16=0,87$ \\
\hline
\end{tabular}

Fuente: elaboración propia

Tras los datos aportados, observamos cómo en todos los casos (excepto para el criterio A en el curso escolar 2019/2020) la diferencia es positiva, lo que se traduce en que las calificaciones siempre fueron mayores (en los cuatro criterios) que las calificaciones medias obtenidas antes de la implementación de la evaluación formativa (figura 4)

Figura 4: Diferencias en las calificaciones medias de los cuatro criterios antes y después de la implementación de la evaluación formativa.

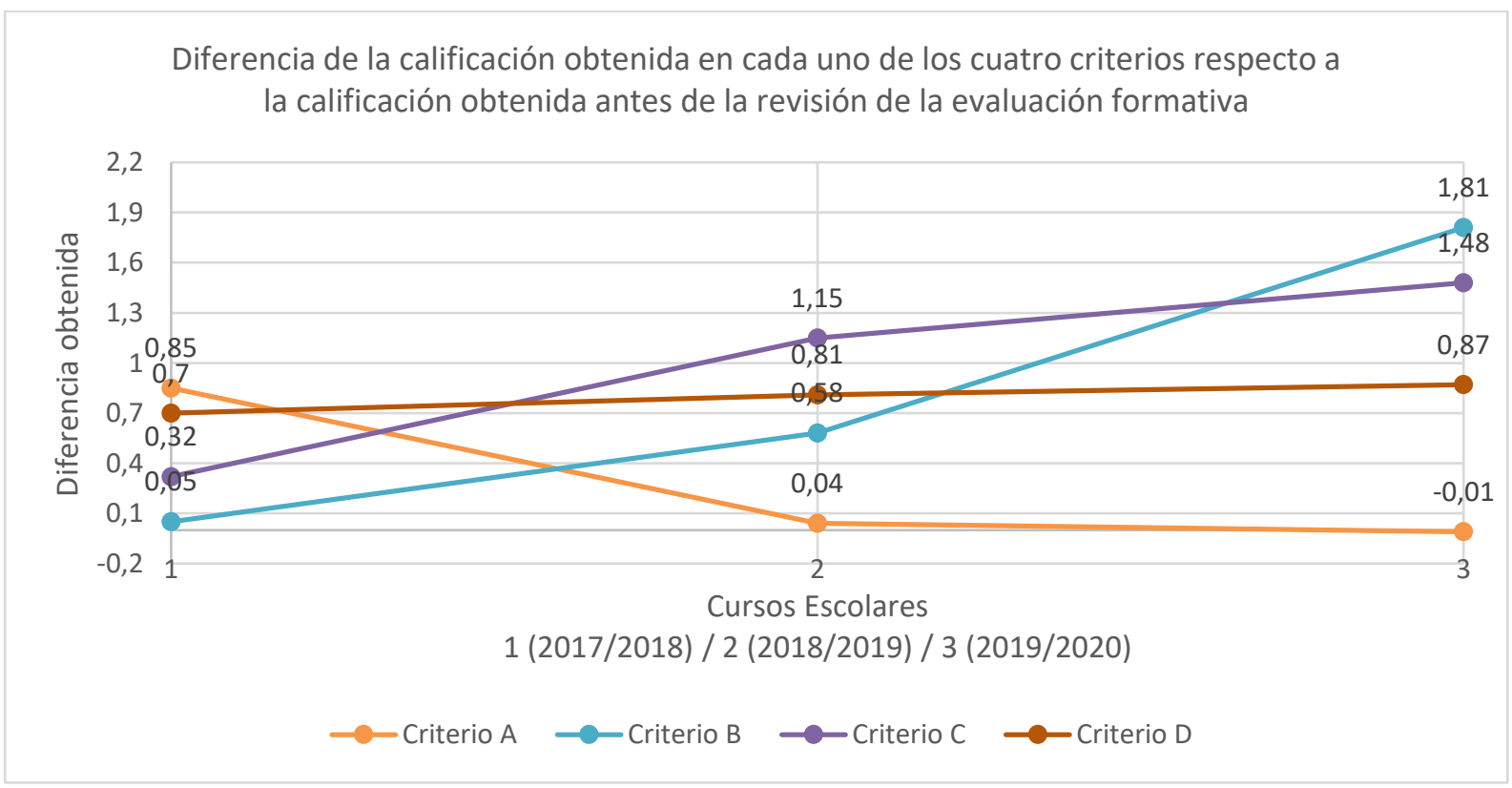

Fuente: elaboración propia 
Destaca el aumento de hasta 1,81 puntos en el criterio B o 1,48 puntos del C, no nos olvidemos que la califiacción de cada criterio puede ser como mucho 8 puntos, por lo que el aumento supone una diferencia muy significativa en la calificación de la nota final de la asignatura de Ciencias.

\subsection{ANOVA}

Con el fin de comprobar si la calificación de los cuatro criterios (A, B, C y D) de Física y Química (Ciencias) es significativamente diferente en los tres cursos escolares estudiados, respecto al anterior a la implementación de la nueva evaluación formativa, se llevó a cabo un Análisis de la Varianza de un factor (tabla 4) mediante el programa informático IBM SPSS.

Tabla 4: ANOVA

\begin{tabular}{|c|c|c|c|c|c|}
\hline & Suma de cuadrados & gl & Media cuadrática & $\mathrm{F}$ & Sig. \\
\hline $\begin{array}{r}\text { Calificación Criterio } A \\
\text { Entre grupos } \\
\text { Dentro de grupos } \\
\text { Total }\end{array}$ & $\begin{array}{r}79,247 \\
1923,472 \\
2002,719\end{array}$ & $\begin{array}{r}3 \\
540 \\
543\end{array}$ & $\begin{array}{r}26,416 \\
3,562\end{array}$ & 7,416 & 0,000 \\
\hline $\begin{array}{r}\text { Calificación Criterio B } \\
\text { Entre grupos } \\
\text { Dentro de grupos } \\
\text { Total }\end{array}$ & $\begin{array}{r}258,829 \\
1657,730 \\
1916,559\end{array}$ & $\begin{array}{r}3 \\
540 \\
543\end{array}$ & $\begin{array}{r}86,276 \\
3,070\end{array}$ & 28,104 & 0,000 \\
\hline $\begin{array}{r}\text { Calificación Criterio } C \\
\text { Entre grupos } \\
\text { Dentro de grupos } \\
\text { Total }\end{array}$ & $\begin{array}{r}185,151 \\
1904,113 \\
2089,265 \\
\end{array}$ & $\begin{array}{r}3 \\
540 \\
543 \\
\end{array}$ & $\begin{array}{r}61,717 \\
3,526\end{array}$ & 61,717 & 0,000 \\
\hline $\begin{array}{r}\text { Calificación Criterio D } \\
\text { Entre grupos } \\
\text { Dentro de grupos } \\
\text { Total }\end{array}$ & $\begin{array}{r}64,768 \\
1450,225 \\
1514,993\end{array}$ & $\begin{array}{r}3 \\
540 \\
543\end{array}$ & $\begin{array}{r}21,589 \\
2,686\end{array}$ & 21,589 & 0,000 \\
\hline
\end{tabular}

Fuente: IBM SPSS Statistics Visor

Como el valor de $\mathrm{F}$ es mayor que 1 podemos afirmar que efectivamente existe un efecto positivo respecto a la variable curso escolar. Además, como P-valor (Sig.) en los cuatro criterios estudiados es de 0,000 , menor del nivel de significación $(0,05)$, se comprueba estadísticamente que los cuatro grupos de cursos escolares analizados son diferentes entre sí.

\subsection{CONTRASTES}

Ahora que sabemos que hay diferencias significativas entre las calificaciones medias de los cuatro criterios. Nos preguntamos: ¿en qué cursos escolares existen dichas diferencias?

Para contestar a esta pregunta realizamos un studio de contrastes donde el curso escolar 2016/2017 se comparará con los tres cursos escolares siguientes para ver si realmente la implementación de la nueva evaluación formative influyó en las caificaciones medias del alumnado (tabla 5).

Tabla 5: Coeficientes de contraste

\begin{tabular}{|c|c|c|c|c|}
\hline \multirow{2}{*}{ Contraste } & \multicolumn{4}{|c|}{ Curso escolar } \\
\cline { 2 - 5 } & $2016 / 2017$ & $2017 / 2018$ & $2018 / 2019$ & $019 / 2020$ \\
\hline 1 & 1 & -1 & 0 & 0 \\
2 & 1 & 0 & -1 & -1 \\
\hline
\end{tabular}

Fuente: IBM SPSS Statistics Visor 
Una vez establecidos los coeficientes de contraste, se procedió a la realización de la prueba t para cada uno de los criterios (A, B, C y D) en cada uno de los cursos escolares que queríamos analizar: desde 2017/2018 hasta 2019/2020 (table 6).

Tabla 6: Prueba t para los contrastes realizados

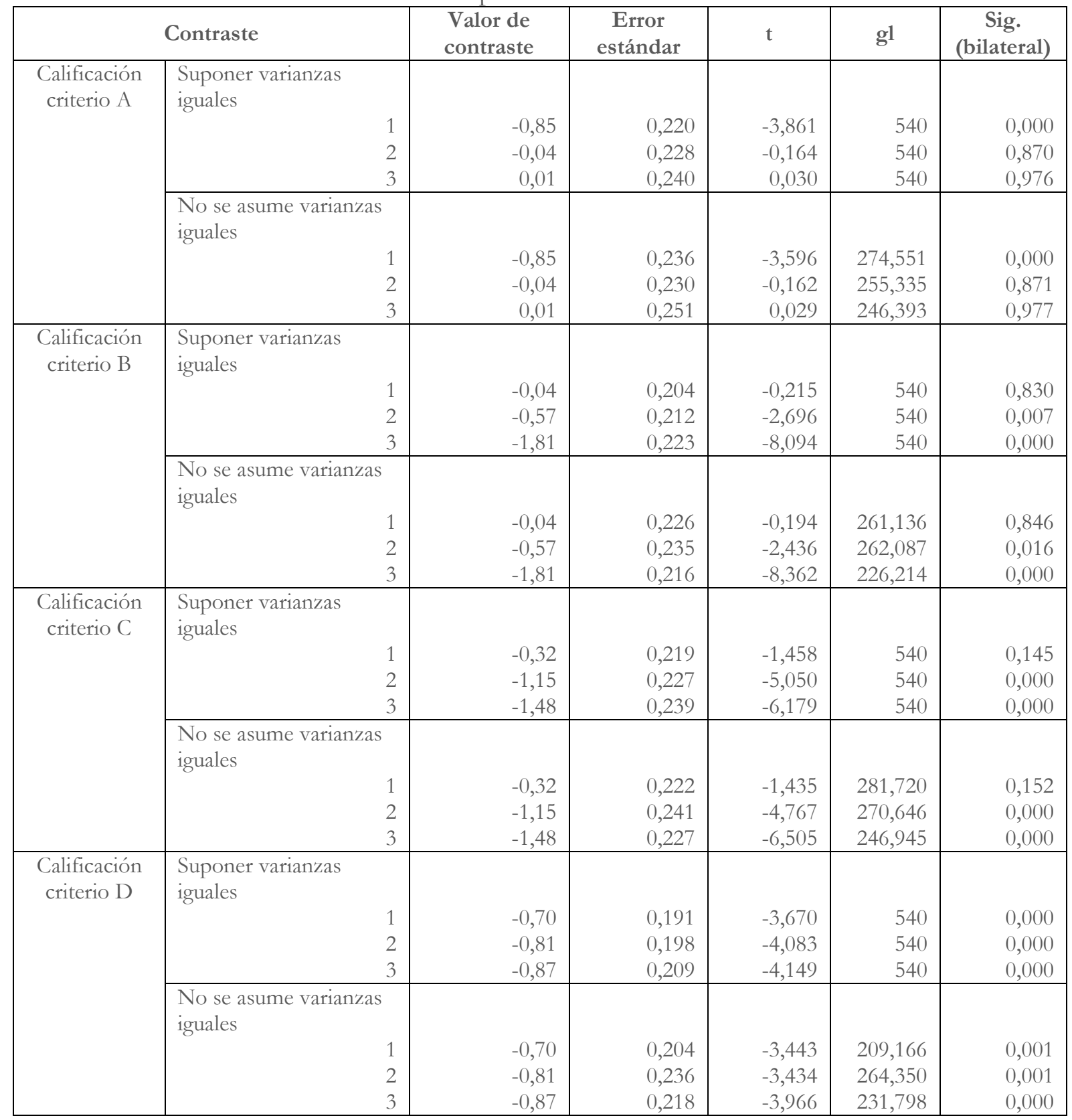

Fuente: IBM SPSS Statistics Visor

\section{DISCUSIÓN}

De los resultados obtenidos se deduce que el período de estudio es suficientemente largo para obtener una serie de conclusiones fiables debido a que se aprecia claramente los patrones que siguen cada uno de los cuatro criterios (A, B, C y D) de la asignatura de Física y Química (Ciencias) en PAI 5. 
Si nos centramos en las medias generales de los cuatro criterios, el criterio en el que los alumnos llevan una mayor calificación es el criterio D $(5,75)$, seguido del $\mathrm{B}(5,30)$, del $\mathrm{C}(4,79)$ y por último el A $(4,34)$. Este reparto de la calificación es muy significativo ya que de los cuatro criterios, el A, es el que require un mayor esfuerzo por parte del alumno, require el estudio del mismo y la comprensión de la materia que el alumno está trabajando, sin embargo, los otros tres criterios (B, $\mathrm{C}$ y D) requieren que se trabajen en mayor profundidad y se potencien una serie de habilidades para que las calificaciones sean exitosas.

Centrándonos en el objetivo de nuestro estudio, con lo que respecta al efecto provocado en las calificaciones de los cuatro criterios de la asignatura de Ciencias, en el PAI, los procedimientos propuestos por Costa y Garmston aplicados a las Ciencias en el aula reflejan un efecto favorable ya que en los cuatro criterios, en los tres cursos siguientes a la mejora de la evaluación formativa, se aprecia que las medias han aumentado (a excepción del criterio A en el curso escolar 2019/2020, donde prácticamente la media ha sido la misma: 4,08 en el curso escolar 2016/2017 y 4,09 en el curso escolar 2017/2018).

Asímismo, este aumento en las medias de las calificaciones de los criterios B, C y D, se aprecia que ha sido progresivo, aumentando las calificaciones a medida que fueron pasando los cursos escolares, tal y como se puede apreciar en la tabla 2 lo cual parece indicar que la profesora fue mejorando la manera en la que se trabajaba la evaluación formativa.

En el informe de la revision del currículo de Ciencias del PAI publicado en el año 2019, uno de los objetivos de mejora que se busca, tras haber implementado y desarrollado el Proyecto de "Programa de los Años Intermedios: el siguiente capítulo" es revisar los objetivos y los criterios de evaluación con el fin de "garantizar que los criterios de evaluación reflejen el equilibrio de las habilidades desarrolladas en Ciencias y que constituyan una herramienta útil para una evaluación sólida y para ayudar a planificar la progresión" (Azzam, Mason, Swope, Ryckaczewska, \& Mansfield, 2018). Dicho objetivo, como se puede apreciar con los datos aportados en este trabajo, no puede alcanzarse si no se planifica adecuadamente una evaluación formativa que garantice que el alumno trabaje, comprenda y aplique los enfoques de aprendizaje que garantice su éxito en las Ciencias.

Por otro lado, según Azzam et al, uno de los hallazgos más significativos que se han encontrado tras la implementación del Proyecto de: "Programa de los Años Intermedios: el siguiente capítulo" es que muchos colegios no implementan el Programa completo, sino que aportan preferencias a determinados componentes del PAI haciendo que el resultado no sea el esperado por los docentes o directivos del centro (Azzam, Mason, Swope, Ryckaczewska, \& Mansfield, 2018).

La mejora progresiva de las tareas sumativas a lo largo del tiempo solamente puede hacerse factible si dentro de la asignatura hay una buena planificación de los enfoques de aprendizaje integrados en la evaluacion formative (figura 5) ya que es evidente la escasez con la que los profesores los trabajan diariamente en el aula (Azzam, Mason, Swope, Ryckaczewska, \& Mansfield, 2018). En el estudio realizado en 2017 a 100 profesores por parte de dichos autores, solamenete la mitad aseguraron que una vez a la semana trabajaban las habilidades de comunicación y colaboración, sin embargo, el resto de los enfoques de aprendizaje (entre los que se encuentra el pensamiento crítico tan importante para la asignatura de Ciencias) reconocieron que casi uno de cada tres no disponía de una planificacion vertical de los mismos y, por lo tanto, no eran potenciados a partir de la evaluación formative. 
Figura 5: Interdependencia entre la evaluación formativa y los resultados finales de la asignatura de Ciencias

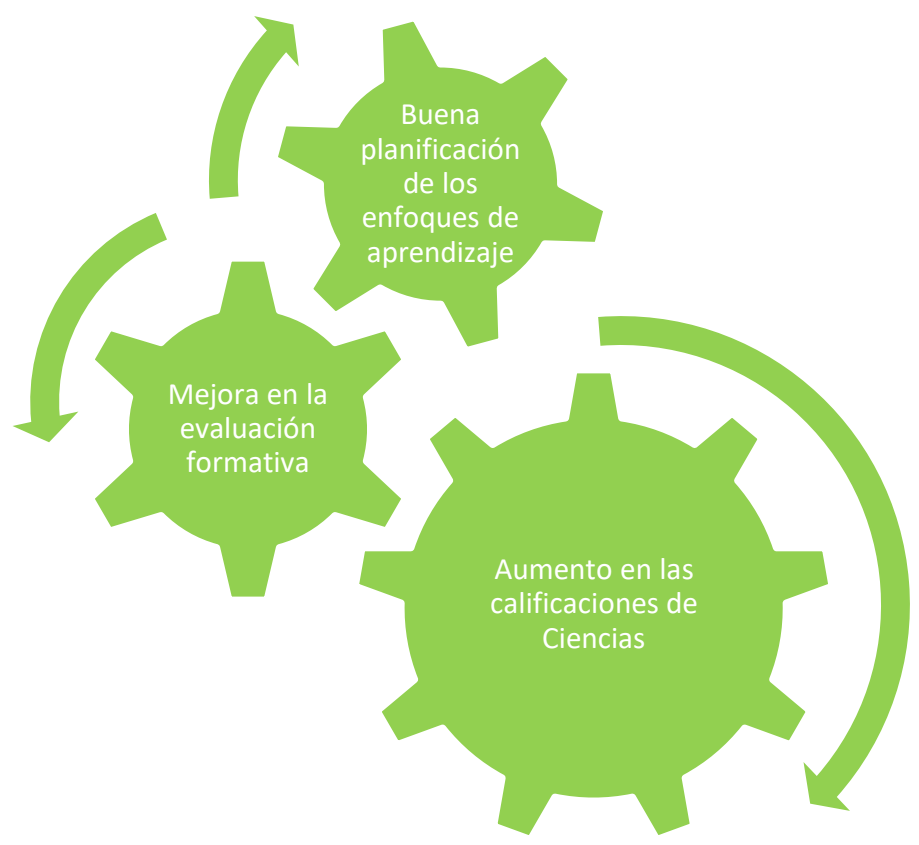

Fuente: Material original

El análisis de la varianza de un criterio (ANOVA) nos permite analizar si hay variación entre las muestras y la variación dentro de las mismas gracias al cálculo de las varianzas. Se emplea el estudio de un criterio porque analiza una variable independiente o factor como es en nuestro caso el curso escolar analizado en nuestro estudio. Siendo este un método adecuado para comparar dos o más medias poblacionales (Reyes, 2004).

Gracias a la ANOVA podemos determinar si las diferencias entre las medias de las calificaciones de los cuatro criterios que hemos obtenido son estadísticamente significativas o no, comparando el P-valor de cada uno de los cuatro criterios con el nivel de significancia $(\alpha)$ empleado que ha sido de 0,05 .

El haber obtenido un P-valor (Sig.) de 0,000 en los cuatro criterios de Ciencias, quiere decir que se rechaza la hipótesis nula o, lo que es lo mismo, que no todas las medias de los cuatro criterios son iguales, ya que $\mathrm{P}$-valor $<\alpha$.

Llegados a este punto, ha quedado demostrado que hay diferencias significativas en función de los cursos escolares pero, ¿realmente hay diferencias significativas en la

calificación de los cuatro criterios de Ciencias en función del cambio en la evaluación formativa?

Para responder a esta pregunta se realizó una prueba de contraste en el que se analizaron los coeficientes de contraste que queríamos trabajar, es decir, se comparó la calificación de los cuatro criterios obtenidos en el curso escolar 2016/2017 con los otros tres cursos escolares implicados en nuestro estudio (2017/2018, 2018/2019 y 2019/2020). 


\section{Prueba t para el criterio A: Conocimiento y comprensión}

Partimos de la base de que, para obtener una buena calificación en este criterio, los alumnos deben dedicar el tiempo necesario para llevar a cabo un estudio de calidad, un estudio no memorístico para poder alcanzar las bandas más altas en los diferentes aspectos del mismo (pueden verse en la parte de introducción del documento), ya que para alcanzarlas se requiere una comprensión profunda de lo estudiado.

En este caso, se observa que en el primer curso escolar en el que se implantó la mejora de la evaluación formativa, el valor p (Sig. Bilateral) fue de 0,000, por lo que rechazaríamos la hipótesis y se concluiría que sí hay diferencias significativas en la calificación obtenida en este criterio por parte de los alumnos, sin embargo, en los dos cursos escolares siguientes (2018/2019 y 2019/2020), los valores obtenidos de p fueron de 0,870 y 0,976 , respectivamente por lo que en este caso, al ser ( $>>0,05)$ no rechazaríamos la hipótesis.

La explicación es clara, la calificación de este criterio viene muy marcada por el estudio del alumno, no tanto por la práctica o la mejora de las habilidades del mismo. De ahí que apreciáramos fluctuaciones en las medias obtenidas de las diferentes promociones.

\section{Prueba t para el criterio B: Indagación y diseño y para el criterio C: Procesamiento y evaluación}

Se analizan conjuntamente estos dos criterios porque el patrón que siguieron ambos fue el mismo. En el primer curso tras la implementación de la evaluación formativa mejorada (2017/2018) en ambos casos se obtuvo un valor p mayor de 0,05 , en concreto: 0,830 y 0,145 , respectivamente, por lo que no podríamos decir que la evaluación formativa tuviera una influencia significativa estadísticamente (aunque sí se observa un aumento en las medias de ambos criterios en el curso escolar comentado respecto al curso escolar tomado como referencia).

Sin embargo, en los siguientes dos cursos analizados, sí aparecen diferencias significativas estadísticamente ( 0,007 y 0,000 para el criterio B y 0,000 en los dos cursos para el criterio C).

La mejora en estos dos criterios es fundamental para que los alumnos puedan comprender realmente los conceptos de la disciplina y la importancia que supone el desarrollar el método científico y cómo éste nos aporta una serie de datos cuyo análisis permite a los científicos explicar aquellos retos que se plantean en sus estudios.

Para poder trabajar bien los aspectos de estos criterios, comentados en la introducción de este documento, es muy importante trabajar las habilidades de comunicación y de pensamiento crítico en la evaluación formativa para que los alumnos puedan extrapolarlas a las tareas sumativas.

Así mismo, tanto el currículum local, Como en el PAI exige a los alumnos, en todos los cursos de Ciencias, realizar un proyecto de investigación donde la elección del tema, su desarrollo y conclusión se lleva a cabo de modo individual. La mejora de estos dos criterios se ha visto reflejado en el desarrollo de dichos proyectos, donde los alumnos realizan proyectos mucho más realistas, con planteamientos de metodología más acertados y evidentemente con una interpretación mucho más correcta y apropiada a la experimentación llevada a cabo. 


\section{Prueba t para el criterio D: Reflexión sobre el impacto de la Ciencia}

Como cabía esperar, este fue el criterio en el que la implementación de una buena evaluación formativa se vería reflejado en las calificaciones obtenidas por el alumnado. El valor t, en los tres contrastes realizados, es decir, en todos los cursos escolares analizados fue de 0,000 , valor menor de 0,05 , por lo que se rechaza la hipótesis nula y sí tiene una influencia positiva en la calificación de este criterio.

Los aspectos trabajados en este criterio (expuestos en el apartado de introducción del documento) son aspectos que implican un fuerte entrenamiento por parte de los alumnos ya que, debido a la influencia de la enseñanza/aprendizaje tradicional, no suelen trabajarse por lo que los alumnos practican menos tareas relacionadas con este criterio a lo largo de toda secundaria por lo que demandan la realización de una gran variedad de tareas formativas con su correspondiente retroalimentación personalizada por parte del profesor.

Como conclusión, queda demostrado que existe una diferencia significativa en el aumento de la calificación obtenida por los alumnos de PAI 5 en la asignatura de Física y Química debido a una enriquecedora evaluación formativa, tal y como se ha mencionado a lo largo de este trabajo. Así como diferencias significativas en la mejora de la comprensión y bandas alcanzadas en los cuatro criterios (A, B , C y D) de la asignatura de Física y Química gracias a la atención y el trabajo individualizado de las habilidades de los alumnos de PAI 5 en las tareas formativas de dicha asignatura.

Como consecuencia, queda patente el hecho de que, no podemos exigirle a nuestro alumnado que "hagan cosas" que nunca han visto o parado a pensar en cómo se podrían hacer. Una buena evaluación formativa implica una enseñanza de calidad, una enseñanza en la que el docente guía al alumno a ir potenciando y adquiriendo aquellas habilidades que necesita para dar respuesta a todos aquellos desafíos de Ciencias que se le plantea. Pero, es más, este andamiaje del aprendizaje por parte del alumno en Ciencias, le permitirá poder seguir construyendo su aprendizaje en cursos futuros, disminuyendo la frustración y el estrés del miedo a la equivocación o al error en una de las disciplinas, junto con las Matemáticas, que suelen temer más los estudiantes. 


\section{BibLIOGRAFÍA}

Azzam, T., Mason, S., Swope, M., Ryckaczewska, A., \& Mansfield, M. (2018). Estudio sobre la implementación y el impacto del "Programa de los Años Intermedios: el siguiente capitulo". Informe II. Claremont: Clarement. Evaluation Center.

Bakieva, M., González Such, J., \& Jornet, J. (2006). SPSS: ANOVA de un factor. Valencia: innovaMIDE / Grupo de Innovación Educativa / Universitat de València.

Costa, A., \& Garmston, R. (2013). Developing Students`Higher-Order Thinking: Supporting SelfDirected Learners. ASCD Express, 8(18).

Franco, V. J. (2012). El enfoque formativo de la evaluación. México, D.F.

IB. (2014). El programa de los Años Intermedios: de los principios a la práctica, para uso a partir de septiembre de 2014 o enero de 2015. Guías Programa de los Años Intermedios, 172.

IB. (2014). Guía de Ciencias para uso a partir de septiembre de 2014 o enero de 2015. Guías del Programe de los Años Intermedios, 79.

Internacional, O. d. (2010). Guía de Ciencias uso a partir de septiembre de 2010 o enero de 2011, según la fecha de inicio del año escolar. Guías Programa de los Años Intermedios, 90.

Osorio Sánchez, K., \& Löpez Mendoza, A. (2014). La retroalimentación formativa en el proceso de Enseñanza-Aprendizaje en Edad Preescolar. Revist a Iberoamericana de Evaluación Educativa, 7(1), 13-30.

Porras, A. M. (2017). Diseño estadístico de experimentos. Granada: Universidad de Granada (España).

Reyes, P. (2004). Análisis de varianza de una vía o dirección (ANOVA 1 VÏA).

Sadler, R. (2010). Beyond feedback: developing student capability in complex appraisal. Assessment \& Evaluation in Higher Education, 35(5), 535-550.

Valle, J. M., Menéndez, M., Manso, J., Garrido, R., \& Thoilliez, B. (2017). Resumen investigación: Implementación y resultados del Programa de los Años Intermedios (PAI) del Bachillerato Internacional (IB) en colegios españoles. Madrid: Universidad autónoma de Madrid / Departamento de investigación del IB. 


\section{ANEXO}

En la tabla que se adjunta a continuación se puede ver detalladamente las calificaciones de los alumnos en cada uno de los cuatro criterios (A, B, C y D) en la asignatura de Física y Química de los alumnos de PAI 5 durante el intervalo en el que se centra el estudio: curso escolar 2016/2017 - curso escolar 2019/2020.

\begin{tabular}{|c|c|c|c|c|c|c|c|c|c|c|c|c|c|c|c|c|}
\hline \multirow{2}{*}{ ID } & \multicolumn{4}{|c|}{$2016 / 2017$} & \multicolumn{4}{|c|}{$2017 / 2018$} & \multicolumn{4}{|c|}{$2018 / 2019$} & \multicolumn{4}{|c|}{$2019 / 2020$} \\
\hline & $\overline{\mathbf{A}}$ & B & $\mathrm{C}$ & D & $\overline{\mathbf{A}}$ & B & $\mathrm{C}$ & D & $\mathbf{A}$ & B & $\mathrm{C}$ & D & $\bar{A}$ & B & $\mathbf{C}$ & D \\
\hline 1 & 4 & 6 & 6 & 7 & 6 & 5 & 6 & 7 & 5 & 3 & 4 & 4 & 4 & 6 & 6 & 6 \\
\hline 2 & 8 & 7 & 7 & 7 & 3 & 4 & 4 & 6 & 6 & 3 & 6 & 3 & 8 & 8 & 8 & 8 \\
\hline 3 & 4 & 7 & 7 & 7 & 1 & 2 & 3 & 5 & 5 & 4 & 4 & 0 & 1 & 5 & 4 & 4 \\
\hline 4 & 1 & 1 & 1 & 1 & 1 & 2 & 2 & 4 & 6 & 5 & 6 & 8 & 3 & 6 & 6 & 8 \\
\hline 5 & 1 & 4 & 4 & 5 & 4 & 5 & 6 & 6 & 6 & 3 & 5 & 5 & 5 & 6 & 6 & 7 \\
\hline 6 & 1 & 4 & 4 & 8 & 6 & 7 & 7 & 7 & 5 & 5 & 5 & 5 & 1 & 5 & 5 & 4 \\
\hline 7 & 1 & 1 & 1 & 1 & 5 & 7 & 7 & 7 & 6 & 6 & 6 & 6 & 1 & 5 & 5 & 5 \\
\hline 8 & 6 & 7 & 7 & 7 & 4 & 7 & 7 & 7 & 4 & 4 & 5 & 7 & 2 & 5 & 5 & 4 \\
\hline 9 & 4 & 5 & 5 & 4 & 2 & 3 & 5 & 5 & 5 & 5 & 5 & 5 & 3 & 6 & 6 & 5 \\
\hline 10 & 2 & 1 & 1 & 1 & 3 & 4 & 3 & 6 & 3 & 4 & 4 & 4 & 1 & 5 & 4 & 4 \\
\hline 11 & 2 & 5 & 5 & 6 & 5 & 6 & 7 & 7 & 4 & 4 & 4 & 5 & 7 & 7 & 6 & 6 \\
\hline 12 & 6 & 7 & 6 & 8 & 3 & 2 & 2 & 7 & 7 & 6 & 7 & 7 & 3 & 7 & 6 & 7 \\
\hline 13 & 7 & 7 & 7 & 7 & 2 & 4 & 4 & 5 & 3 & 5 & 4 & 1 & 4 & 6 & 6 & 6 \\
\hline 14 & 5 & 6 & 6 & 6 & 5 & 6 & 2 & 6 & 5 & 4 & 4 & 3 & 7 & 8 & 8 & 7 \\
\hline 15 & 1 & 3 & 3 & 1 & 3 & 3 & 3 & 6 & 3 & 2 & 3 & 3 & 3 & 5 & 4 & 6 \\
\hline 16 & 1 & 3 & 3 & 1 & 7 & 5 & 3 & 7 & 7 & 7 & 7 & 8 & 5 & 7 & 6 & 4 \\
\hline 17 & 7 & 7 & 7 & 8 & 4 & 6 & 5 & 7 & 7 & 7 & 7 & 8 & 8 & 7 & 6 & 6 \\
\hline 18 & 6 & 6 & 6 & 7 & 4 & 4 & 3 & 6 & 7 & 7 & 6 & 5 & 6 & 7 & 6 & 8 \\
\hline 19 & 5 & 6 & 6 & 6 & 7 & 8 & 8 & 8 & 6 & 7 & 7 & 7 & 8 & 8 & 8 & 8 \\
\hline 20 & 7 & 6 & 6 & 6 & 8 & 8 & 8 & 8 & 5 & 7 & 6 & 6 & 8 & 7 & 6 & 8 \\
\hline 21 & 2 & 1 & 1 & 1 & 5 & 2 & 4 & 6 & 5 & 3 & 4 & 0 & 3 & 7 & 6 & 5 \\
\hline 22 & 2 & 3 & 3 & 2 & 6 & 5 & 5 & 7 & 3 & 3 & 3 & 4 & 4 & 6 & 6 & 7 \\
\hline 23 & 1 & 3 & 3 & 3 & 2 & 3 & 3 & 6 & 5 & 4 & 5 & 3 & 1 & 5 & 4 & 4 \\
\hline 24 & 7 & 7 & 7 & 8 & 2 & 3 & 2 & 5 & 4 & 4 & 4 & 4 & 2 & 6 & 5 & 5 \\
\hline 25 & 6 & 6 & 6 & 7 & 4 & 4 & 3 & 7 & 4 & 3 & 4 & 5 & 8 & 8 & 7 & 8 \\
\hline 26 & 3 & 5 & 4 & 7 & 6 & 6 & 6 & 7 & 8 & 8 & 8 & 8 & 3 & 5 & 5 & 5 \\
\hline 27 & 2 & 3 & 4 & 5 & 5 & 5 & 5 & 6 & 5 & 6 & 6 & 7 & 7 & 7 & 6 & 5 \\
\hline 28 & 4 & 5 & 5 & 4 & 4 & 3 & 1 & 7 & 2 & 4 & 4 & 2 & 3 & 6 & 5 & 6 \\
\hline 29 & 8 & 7 & 7 & 8 & 1 & 3 & 3 & 6 & 7 & 8 & 7 & 7 & 6 & 7 & 7 & 6 \\
\hline 30 & 3 & 3 & 3 & 5 & 5 & 6 & 7 & 7 & 5 & 5 & 5 & 3 & 1 & 4 & 3 & 4 \\
\hline 31 & 3 & 3 & 3 & 5 & 4 & 4 & 3 & 5 & 6 & 6 & 6 & 7 & 1 & 3 & 4 & 4 \\
\hline 32 & 7 & 7 & 7 & 7 & 3 & 5 & 7 & 6 & 4 & 4 & 4 & 3 & 4 & 7 & 6 & 6 \\
\hline 33 & 2 & 4 & 3 & 5 & 1 & 3 & 2 & 6 & 6 & 5 & 6 & 6 & 7 & 7 & 7 & 6 \\
\hline 34 & 8 & 8 & 8 & 8 & 6 & 6 & 7 & 7 & 2 & 2 & 2 & 1 & 8 & 7 & 7 & 8 \\
\hline 35 & 6 & 5 & 5 & 5 & 3 & 3 & 3 & 6 & 2 & 3 & 3 & 3 & 5 & 7 & 6 & 8 \\
\hline 36 & 3 & 4 & 4 & 4 & 5 & 8 & 7 & 7 & 6 & 6 & 7 & 8 & 3 & 6 & 5 & 5 \\
\hline 37 & 6 & 6 & 6 & 6 & 6 & 6 & 8 & 7 & 4 & 4 & 5 & 5 & 1 & 5 & 4 & 4 \\
\hline 38 & 6 & 7 & 6 & 6 & 2 & 3 & 3 & 5 & 6 & 5 & 6 & 6 & 3 & 6 & 5 & 4 \\
\hline 39 & 3 & 1 & 1 & 2 & 2 & 1 & 2 & 5 & 6 & 4 & 5 & 4 & 4 & 6 & 6 & 6 \\
\hline 40 & 6 & 6 & 6 & 7 & 3 & 4 & 4 & 6 & 5 & 3 & 5 & 5 & 4 & 6 & 6 & 5 \\
\hline 41 & 6 & 4 & 4 & 2 & 5 & 3 & 2 & 6 & 3 & 5 & 4 & 5 & 7 & 7 & 6 & 7 \\
\hline 42 & 6 & 5 & 5 & 5 & 4 & 4 & 5 & 6 & 3 & 0 & 0 & 1 & 7 & 7 & 7 & 8 \\
\hline 43 & 1 & 5 & 5 & 4 & 2 & 5 & 4 & 6 & 6 & 6 & 6 & 5 & 6 & 6 & 6 & 6 \\
\hline 44 & 5 & 5 & 4 & 4 & 3 & 6 & 6 & 7 & 5 & 4 & 5 & 4 & 8 & 7 & 7 & 8 \\
\hline 45 & 2 & 5 & 5 & 4 & 8 & 8 & 8 & 8 & 4 & 4 & 4 & 3 & 1 & 5 & 5 & 3 \\
\hline 46 & 2 & 3 & 3 & 2 & 6 & 4 & 4 & 6 & 6 & 5 & 6 & 4 & 4 & 7 & 6 & 6 \\
\hline 47 & 3 & 5 & 4 & 5 & 4 & 5 & 3 & 7 & 6 & 4 & 5 & 4 & 1 & 5 & 4 & 3 \\
\hline
\end{tabular}




\begin{tabular}{|c|c|c|c|c|c|c|c|c|c|c|c|c|c|c|c|c|}
\hline 48 & 5 & 7 & 4 & 6 & 3 & 6 & 6 & 6 & 5 & 5 & 2 & 6 & 3 & 6 & 5 & 6 \\
\hline 49 & 5 & 7 & 7 & 7 & 4 & 3 & 3 & 6 & 3 & 6 & 5 & 6 & 6 & 7 & 6 & 7 \\
\hline 50 & 4 & 7 & 6 & 6 & 1 & 2 & 2 & 4 & 4 & 6 & 8 & 6 & 4 & 7 & 6 & 7 \\
\hline 51 & 1 & 4 & 2 & 2 & 3 & 4 & 5 & 6 & 5 & 7 & 3 & 6 & 1 & 5 & 5 & 5 \\
\hline 52 & 3 & 4 & 2 & 2 & 6 & 7 & 8 & 7 & 3 & 6 & 7 & 6 & 3 & 6 & 6 & 6 \\
\hline 53 & 6 & 6 & 1 & 6 & 5 & 7 & 7 & 7 & 3 & 7 & 8 & 6 & 3 & 6 & 6 & 5 \\
\hline 54 & 1 & 4 & 1 & 2 & 7 & 6 & 5 & 5 & 5 & 7 & 7 & 7 & 4 & 7 & 6 & 5 \\
\hline 55 & 7 & 7 & 6 & 7 & 7 & 1 & 4 & 7 & 4 & 7 & 6 & 6 & 3 & 7 & 6 & 6 \\
\hline 56 & 3 & 5 & 5 & 6 & 3 & 6 & 6 & 5 & 2 & 5 & 7 & 6 & 7 & 7 & 7 & 5 \\
\hline 57 & 2 & 5 & 3 & 5 & 4 & 5 & 3 & 2 & 3 & 7 & 7 & 6 & 4 & 8 & 4 & 6 \\
\hline 58 & 3 & 5 & 4 & 5 & 8 & 4 & 7 & 6 & 4 & 7 & 3 & 6 & 7 & 8 & 8 & 8 \\
\hline 59 & 7 & 7 & 7 & 8 & 8 & 6 & 6 & 8 & 4 & 8 & 8 & 7 & 4 & 5 & 2 & 5 \\
\hline 60 & 7 & 7 & 7 & 8 & 6 & 6 & 5 & 6 & 3 & 4 & 7 & 6 & 3 & 8 & 6 & 6 \\
\hline 61 & 3 & 6 & 3 & 6 & 8 & 7 & 5 & 3 & 4 & 7 & 5 & 6 & 3 & 8 & 6 & 8 \\
\hline 62 & 1 & 4 & 2 & 4 & 5 & 4 & 3 & 4 & 1 & 6 & 6 & 6 & 3 & 7 & 3 & 6 \\
\hline 63 & 1 & 4 & 1 & 2 & 4 & 1 & 2 & 8 & 4 & 8 & 6 & 7 & 4 & 8 & 6 & 8 \\
\hline 64 & 7 & 7 & 8 & 8 & 7 & 3 & 3 & 5 & 4 & 7 & 8 & 6 & 3 & 6 & 2 & 6 \\
\hline 65 & 4 & 5 & 5 & 7 & 6 & 2 & 4 & 3 & 5 & 8 & 7 & 6 & 4 & 8 & 3 & 6 \\
\hline 66 & 6 & 7 & 7 & 7 & 7 & 3 & 2 & 4 & 3 & 8 & 7 & 6 & 4 & 5 & 5 & 6 \\
\hline 67 & 7 & 7 & 6 & 8 & 5 & 5 & 1 & 4 & 2 & 4 & 1 & 6 & 5 & 8 & 7 & 6 \\
\hline 68 & 1 & 4 & 1 & 1 & 6 & 2 & 2 & 5 & 4 & 7 & 3 & 6 & 4 & 8 & 8 & 6 \\
\hline 69 & 3 & 5 & 3 & 5 & 8 & 3 & 5 & 5 & 4 & 4 & 6 & 6 & 3 & 7 & 3 & 6 \\
\hline 70 & 4 & 7 & 6 & 7 & 8 & 4 & 5 & 7 & 3 & 4 & 2 & 6 & 4 & 8 & 7 & 6 \\
\hline 71 & 5 & 8 & 5 & 8 & 8 & 3 & 8 & 5 & 5 & 5 & 8 & 5 & 5 & 8 & 5 & 6 \\
\hline 72 & 4 & 7 & 3 & 5 & 8 & 8 & 7 & 7 & 8 & 8 & 8 & 8 & 4 & 7 & 7 & 8 \\
\hline 73 & 2 & 7 & 2 & 4 & 8 & 8 & 8 & 8 & 3 & 5 & 3 & 6 & 5 & 8 & 6 & 8 \\
\hline 74 & 5 & 8 & 6 & 8 & 5 & 3 & 1 & 5 & 5 & 6 & 6 & 7 & 4 & 7 & 8 & 8 \\
\hline 75 & 7 & 8 & 6 & 8 & 8 & 5 & 2 & 7 & 5 & 8 & 8 & 7 & 6 & 8 & 8 & 8 \\
\hline 76 & 3 & 5 & 2 & 6 & 6 & 6 & 4 & 6 & 4 & 5 & 5 & 6 & 4 & 8 & 8 & 6 \\
\hline 77 & 5 & 7 & 5 & 8 & 4 & 4 & 4 & 5 & 5 & 8 & 8 & 6 & 5 & 6 & 7 & 6 \\
\hline 78 & 3 & 7 & 3 & 6 & 8 & 3 & 3 & 4 & 4 & 4 & 7 & 6 & 5 & 8 & 8 & 6 \\
\hline 79 & 5 & 8 & 6 & 6 & 8 & 2 & 5 & 4 & 5 & 5 & 7 & 6 & 3 & 1 & 1 & 5 \\
\hline 80 & 5 & 5 & 5 & 7 & 7 & 6 & 4 & 7 & 4 & 7 & 4 & 6 & 1 & 8 & 4 & 6 \\
\hline 81 & 2 & 6 & 3 & 4 & 5 & 4 & 2 & 6 & 6 & 8 & 4 & 7 & 4 & 8 & 6 & 6 \\
\hline 82 & 3 & 7 & 6 & 7 & 6 & 4 & 3 & 3 & 4 & 7 & 7 & 6 & 5 & 7 & 5 & 5 \\
\hline 83 & 6 & 7 & 5 & 7 & 8 & 4 & 6 & 7 & 6 & 8 & 6 & 7 & 5 & 8 & 7 & 6 \\
\hline 84 & 1 & 2 & 1 & 4 & 3 & 2 & 1 & 4 & 4 & 4 & 2 & 6 & 3 & 7 & 6 & 6 \\
\hline 85 & 5 & 7 & 3 & 5 & 4 & 7 & 5 & 5 & 3 & 4 & 5 & 6 & 4 & 8 & 5 & 6 \\
\hline 86 & 2 & 5 & 3 & 3 & 2 & 2 & 1 & 3 & 2 & 6 & 1 & 6 & 5 & 7 & 4 & 6 \\
\hline 87 & 4 & 6 & 3 & 6 & 3 & 5 & 5 & 7 & 3 & 7 & 3 & 6 & 3 & 7 & 3 & 5 \\
\hline 88 & 4 & 7 & 1 & 2 & 4 & 4 & 4 & 6 & 3 & 5 & 7 & 6 & 3 & 7 & 7 & 8 \\
\hline 89 & 3 & 6 & 5 & 5 & 8 & 5 & 4 & 4 & 3 & 7 & 3 & 5 & 4 & 8 & 5 & 6 \\
\hline 90 & 3 & 6 & 3 & 4 & 5 & 4 & 5 & 7 & 4 & 4 & 6 & 5 & 5 & 7 & 8 & 8 \\
\hline 91 & 2 & 3 & 1 & 0 & 6 & 1 & 1 & 4 & 4 & 4 & 1 & 6 & 4 & 7 & 6 & 6 \\
\hline 92 & 3 & 5 & 4 & 2 & 2 & 3 & 1 & 5 & 4 & 6 & 8 & 8 & 3 & 6 & 3 & 4 \\
\hline 93 & 5 & 3 & 4 & 8 & 5 & 6 & 2 & 5 & 1 & 7 & 7 & 8 & 5 & 5 & 5 & 5 \\
\hline 94 & 7 & 7 & 6 & 5 & 5 & 5 & 4 & 6 & 1 & 4 & 4 & 8 & 3 & 7 & 1 & 6 \\
\hline 95 & 7 & 5 & 4 & 8 & 6 & 5 & 5 & 6 & 6 & 7 & 8 & 8 & 4 & 6 & 7 & 5 \\
\hline 96 & 1 & 1 & 2 & 4 & 8 & 5 & 2 & 4 & 7 & 3 & 7 & 7 & 4 & 7 & 7 & 8 \\
\hline 97 & 4 & 2 & 1 & 3 & 5 & 3 & 2 & 6 & 3 & 6 & 3 & 8 & 4 & 6 & 8 & 6 \\
\hline 98 & 4 & 0 & 1 & 3 & 8 & 8 & 8 & 7 & 4 & 5 & 5 & 8 & 3 & 7 & 7 & 8 \\
\hline 99 & 1 & 2 & 1 & 3 & 7 & 7 & 3 & 5 & 2 & 3 & 6 & 8 & 3 & 5 & 6 & 6 \\
\hline 100 & 6 & 7 & 6 & 6 & 5 & 5 & 6 & 6 & 1 & 5 & 6 & 8 & 5 & 7 & 7 & 8 \\
\hline 101 & 6 & 3 & 2 & 5 & 5 & 4 & 3 & 5 & 3 & 5 & 4 & 8 & 4 & 4 & 1 & 4 \\
\hline 102 & 2 & 0 & 4 & 3 & 5 & 2 & 1 & 3 & 4 & 7 & 7 & 6 & 4 & 7 & 6 & 8 \\
\hline 103 & 3 & 3 & 4 & 5 & 2 & 2 & 3 & 3 & 7 & 8 & 8 & 8 & 5 & 5 & 1 & 5 \\
\hline 104 & 7 & 3 & 3 & 8 & 6 & 6 & 5 & 6 & 2 & 6 & 3 & 8 & 4 & 6 & 4 & 6 \\
\hline
\end{tabular}




\begin{tabular}{|c|c|c|c|c|c|c|c|c|c|c|c|c|c|c|c|c|}
\hline 105 & 5 & 4 & 2 & 4 & 7 & 8 & 7 & 8 & 2 & 6 & 6 & 7 & 5 & 8 & 5 & 5 \\
\hline 106 & 6 & 1 & 2 & 6 & 7 & 7 & 4 & 5 & 2 & 3 & 4 & 6 & 3 & 8 & 6 & 6 \\
\hline 107 & 1 & 2 & 1 & 3 & 6 & 6 & 6 & 6 & 6 & 7 & 8 & 8 & 2 & 5 & 5 & 6 \\
\hline 108 & 1 & 2 & 1 & 2 & 4 & 6 & 5 & 6 & 4 & 5 & 7 & 7 & 4 & 8 & 5 & 6 \\
\hline 109 & 8 & 7 & 5 & 5 & 5 & 6 & 4 & 5 & 4 & 8 & 8 & 8 & 3 & 8 & 7 & 8 \\
\hline 110 & 2 & 3 & 3 & 7 & 4 & 6 & 3 & 6 & 5 & 7 & 8 & 8 & 6 & 6 & 6 & 5 \\
\hline 111 & 5 & 5 & 3 & 3 & 6 & 6 & 6 & 7 & 1 & 4 & 5 & 5 & 4 & 7 & 7 & 5 \\
\hline 112 & 7 & 6 & 4 & 8 & 7 & 6 & 6 & 6 & 2 & 7 & 2 & 5 & 3 & 8 & 6 & 6 \\
\hline 113 & 5 & 0 & 1 & 2 & 3 & 6 & 5 & 7 & 4 & 4 & 3 & 8 & & & & \\
\hline 114 & 1 & 1 & 1 & 3 & 7 & 7 & 6 & 8 & 3 & 6 & 7 & 8 & & & & \\
\hline 115 & 6 & 5 & 4 & 7 & 6 & 5 & 4 & 6 & 3 & 3 & 7 & 6 & & & & \\
\hline 116 & 7 & 7 & 7 & 7 & 6 & 4 & 3 & 6 & 8 & 8 & 8 & 8 & & & & \\
\hline 117 & 5 & 3 & 3 & 4 & 5 & 4 & 4 & 5 & 1 & 1 & 2 & 7 & & & & \\
\hline 118 & 4 & 3 & 3 & 5 & 4 & 5 & 5 & 5 & 7 & 7 & 8 & 7 & & & & \\
\hline 119 & 7 & 4 & 5 & 8 & 5 & 6 & 4 & 5 & 5 & 8 & 7 & 8 & & & & \\
\hline 120 & 6 & 8 & 6 & 7 & 6 & 6 & 5 & 6 & 2 & 4 & 2 & 7 & & & & \\
\hline 121 & 1 & 2 & 3 & 4 & 6 & 7 & 5 & 6 & 5 & 6 & 6 & 7 & & & & \\
\hline 122 & 5 & 3 & 5 & 5 & 6 & 6 & 5 & 7 & 3 & 3 & 3 & 7 & & & & \\
\hline 123 & 3 & 4 & 3 & 5 & 5 & 4 & 6 & 6 & 2 & 5 & 1 & 8 & & & & \\
\hline 124 & 7 & 8 & 6 & 7 & 7 & 7 & 7 & 7 & 3 & 1 & 5 & 7 & & & & \\
\hline 125 & 5 & 4 & 6 & 5 & 8 & 8 & 8 & 7 & 5 & 6 & 5 & 6 & & & & \\
\hline 126 & 2 & 3 & 5 & 4 & 4 & 5 & 3 & 4 & 4 & 6 & 1 & 8 & & & & \\
\hline 127 & 6 & 6 & 6 & 7 & 7 & 6 & 5 & 6 & 5 & 5 & 8 & 8 & & & & \\
\hline 128 & 7 & 7 & 4 & 7 & 3 & 6 & 5 & 6 & 5 & 6 & 5 & 5 & & & & \\
\hline 129 & 1 & 0 & 1 & 3 & 4 & 6 & 4 & 6 & 4 & 7 & 5 & 7 & & & & \\
\hline 130 & 6 & 7 & 6 & 6 & 5 & 4 & 3 & 6 & 3 & 7 & 7 & 7 & & & & \\
\hline 131 & 3 & 3 & 2 & 4 & 6 & 6 & 5 & 6 & 1 & 8 & 5 & 5 & & & & \\
\hline 132 & 5 & 3 & 5 & 6 & 6 & 5 & 5 & 6 & 3 & 5 & 7 & 6 & & & & \\
\hline 133 & 2 & 3 & 2 & 8 & 6 & 5 & 5 & 6 & 5 & 4 & 8 & 8 & & & & \\
\hline 134 & 5 & 3 & 5 & 6 & 3 & 4 & 4 & 6 & 1 & 5 & 1 & 8 & & & & \\
\hline 135 & 4 & 2 & 6 & 5 & 7 & 7 & 7 & 7 & 5 & 6 & 6 & 8 & & & & \\
\hline 136 & 2 & 3 & 5 & 5 & 3 & 4 & 3 & 6 & 1 & 4 & 2 & 8 & & & & \\
\hline 137 & 2 & 3 & 5 & 5 & 3 & 5 & 5 & 6 & & & & & & & & \\
\hline 138 & & & & & 3 & 4 & 3 & 6 & & & & & & & & \\
\hline 139 & & & & & 4 & 6 & 6 & 6 & & & & & & & & \\
\hline 140 & & & & & 4 & 4 & 4 & 5 & & & & & & & & \\
\hline 141 & & & & & 8 & 7 & 7 & 8 & & & & & & & & \\
\hline 142 & & & & & 5 & 6 & 6 & 6 & & & & & & & & \\
\hline 143 & & & & & 3 & 4 & 4 & 4 & & & & & & & & \\
\hline 144 & & & & & 3 & 4 & 3 & 6 & & & & & & & & \\
\hline 145 & & & & & 4 & 6 & 5 & 6 & & & & & & & & \\
\hline 146 & & & & & 6 & 5 & 5 & 6 & & & & & & & & \\
\hline 147 & & & & & 3 & 4 & 4 & 5 & & & & & & & & \\
\hline 148 & & & & & 3 & 5 & 5 & 7 & & & & & & & & \\
\hline 149 & & & & & 3 & 5 & 3 & 5 & & & & & & & & \\
\hline 150 & & & & & 8 & 7 & 7 & 7 & & & & & & & & \\
\hline 151 & & & & & 5 & 3 & 3 & 5 & & & & & & & & \\
\hline 152 & & & & & 5 & 5 & 5 & 7 & & & & & & & & \\
\hline 153 & & & & & 3 & 5 & 4 & 5 & & & & & & & & \\
\hline 154 & & & & & 4 & 3 & 3 & 7 & & & & & & & & \\
\hline 155 & & & & & 3 & 6 & 3 & 6 & & & & & & & & \\
\hline 156 & & & & & 4 & 6 & 5 & 6 & & & & & & & & \\
\hline 157 & & & & & 6 & 7 & 7 & 6 & & & & & & & & \\
\hline 158 & & & & & 5 & 6 & 6 & 7 & & & & & & & & \\
\hline 159 & & & & & 4 & 4 & 4 & 5 & & & & & & & & \\
\hline
\end{tabular}




\section{SOBRE LA AUTORA}

\section{Mónica Azpilicueta Amorín (Colegiada n $\left.{ }^{0} 18429-X\right)$}

Licenciada en Biología con grado por la Universidad de Vigo en la rama Fundamental y Sanitaria. Comienzo de la etapa docente desde el curso 1999/2000 en el centro SEK International School Atlántico impartiendo clases de Ciencias (Secundaria y Bachillerato), Tecnología (Secundaria) y Sistemas Ambientales y Sociedades (Programa Diploma), así como actividades extraescolares de Robótica a alumnos de Primaria y Secundaria hasta la actualidad.

Puestos relacionados con el Bachillerato Internacional (IB):

> Coordinadora PAI desde el curso escolar 2009/2010 en el SEK International School hasta la actualidad.

$>$ Experto en Educación Internacional / International Baccalaureate certificate in teaching and learning por la Universidad Camilo José Cela en junio 2015.

$>$ Consultora y Miembro de equipo visitante del PAI desde el curso escolar desde marzo 2015.

Examinadora de Proyectos Personales del PAI desde 2017.

Ponencias impartidas en las Conferencias Regionales del IB:

- Haya del 24 al 27 de octubre de 2013. Título: iTEC meets New Experiences for Engaging Learning.

- Roma del 16 al 19 de octubre de 2014. Título: El libro, un poderoso recurso para el conocimiento.

- Barcelona del 6 al 8 de octubre de 2016. Título: La creatividad y la tecnología al servicio de la comunidad.

Contact information: SEK International School Atlántico. Calle Illa de Arousa 4, Boa Vista, Poio, Pontevedra, C.P. 36005. (monica.azpilicueta@sek.es). 